\title{
Sulfonic Containing Polymer Bead Synthesized through Inverse Suspension Polymerization and Its Characteristics for Esterification Catalyst
}

\author{
Cengliang Shan, Ye Wang, Jun Nie, and Yong He 10 \\ State Key Lab of Chemical Resource Engineering and Key Laboratory of Carbon Fiber and Functional Polymers, \\ Ministry of Education, Beijing University of Chemical Technology, Beijing 100029, China \\ Correspondence should be addressed to Yong He; heyong@mail.buct.edu.cn
}

Received 12 December 2018; Revised 15 March 2019; Accepted 11 April 2019; Published 2 May 2019

Academic Editor: Leonard D. Tijing

Copyright (c) 2019 Cengliang Shan et al. This is an open access article distributed under the Creative Commons Attribution License, which permits unrestricted use, distribution, and reproduction in any medium, provided the original work is properly cited.

\begin{abstract}
The sulfonic containing polymer bead was synthesized using sodium p-styrenesulfonate (SSS) and N,N'-methylenebisacrylamide (MBA) through inverse suspension polymerization and evaluated as catalyst for esterification of of $n$-octanol and acrylic acid. The influence of some principal factors, such as combination dispersant, crosslink agent content, posttreatment methods, and porogen types, was investigated in detail. The results showed that the morphology and characteristics of polymer beads were controllable. The polymer beads with $20 \mathrm{wt} \%$ crosslink agent showed the best catalysis ability achieving almost $96 \%$ esterification conversion at the first time and $80 \%$ after 5 cycles.
\end{abstract}

\section{Introduction}

Ester, as one of most important industrial chemicals, is commercially synthesized by esterification or transesterification usually employing strong mineral acid as catalyst, which could deteriorate the esterification product quality in some degree. Due to the increasing demand of environmental protection, the problem of the disposal of catalyst waste has gradually attracted people's attention and stimulated the searching for recyclable solid catalyst to replace conventional toxic and corrosive acid $[1,2]$. Therefore, there were many attempts with different mechanism and structure reported, such as heteropoly acids [3], zeolites [4], layered material [5], ion exchange resin [6], or enzyme [7, 8]. Among them, ion exchange resin undoubtedly received more and more attention, because it is one of the most widely used materials in many applications for their excellent performance and possesses obvious engineering benefits of easy separation, noncorrosivity, and reusability $[9,10]$.

Sert [11] studied three different ion exchange resins, Amberlyst 15, Amberlyst 131, and Dowex 50Wx-400, as catalyst for the esterification of acrylic acid and n-butanol. They investigated the effects of different variables, temperature, molar ratio of alcohol to acid, stirrer speed, and catalyst loading on the reaction rate and concluded that Amberlyst 131 is the most efficient catalyst giving the maximum conversion of acrylic acid. Styrene-divinylbenzene (St-DVB) copolymer bead was firstly synthesized in the 1960s by suspension polymerization and could be donated sulfonic group $\left(\mathrm{SO}_{3} \mathrm{H}\right)$ through sulfonation to form ion exchange resin [12]. It was proved that the catalysis efficiency of sulfonated polydivinylbenzene (polyDVB- $\mathrm{SO}_{3} \mathrm{H}$ ) can be analogized to commercial ion-exchange resins (Amberlyst 35 and Amberlyst 36) by Aguiar [13] and it was revealed that higher sulfonation, higher ion capacity, and lower carboxylation were desirable [14]. All of the abovementioned systems have to involve a complicated sulfonation process to modify the polymer, which could lead to oxidation, degradation, difficulty in controlling the morphology, or active group ratio, due to long time contact with sulfuric and nitric acid. Therefore, the polymerization of sulfonic containing monomer would be a solution to overcome these problems and to prepare higher quality catalyst beads with good catalyzed ability, stability, and probability to adjust the morphology.

Inverse suspension polymerization is one of the best methods to form polymer particle from water soluble 
monomer with high stability, narrow particle size distribution, fast process rate, and high conversion $[15,16]$. It is reasonable to be adopted in preparation of new polymer beads catalyst with sulfonic containing monomer.

In this work, we aimed to design and prepare a kind of pore size and morphology controllable, reusable polymer bead catalyst for esterification using sulfonic containing monomer through inverse suspension polymerization. The influence of several key factors for the polymer beads catalyst ability, particle size, and morphology, such as the dispersant, crosslink agent, porogen, and preparing condition, was investigated. The catalysis ability regarding the esterification of acrylic acid and $n$-octanol was evaluated.

\section{Experiment}

2.1. Material. Sodium p-styrene sulfonate (SSS) was obtained from Shandong Star Alliance Biotechnology Co. (Shandong, China). N, $\mathrm{N}^{\prime}$-methylenebisacrylamide (MBA) and p-hydroxyanisole were supplied by Tianjin Guangfu Fine Chemical Institute (Tianjin, China). Persulfate, liquid paraffin, acrylic acid, n-octanol, span60, and tween60 were purchased from Tianjin Fuchen Chemical Reagent Factory (Tianjin, China). Propanediol, n-butanol, 1,4-butanediol, and polyethylene glycol (PEG200, PEG400, PEG600) were supplied by Beijing Chemical Works (Beijing, China). All regents were of analytical grade and used without further purification.

2.2. Polymer Beads Characterization. The Fourier transform infrared spectra (FT-IR) were recorded on Nicolet 5700 (Nicolet Instrument, Thermo Company, USA). Samples were prepared with $\mathrm{KBr}$ powder and pressed into flake.

The sulfonic group content was represented from the total amount of sulfur as measured by elemental analysis (CHNS Mode, Elementar Analysensysteme GmbH Company, German) through burnt polymer at high temperature.

The titration was used to measure ion exchange capacity. The pretreated polymer beads with acid were dried by freezedrying for a whole day, and $40 \mathrm{mg}$ beads were taken, suspended in $10 \mathrm{~mL}$ deionized water, and titrated with 0.1 $\mathrm{mol} / \mathrm{L}$ standard $\mathrm{NaOH}$ aqueous solution in the presence of a phenolphthalein indicator [17-19].

Laser scattering particle size distribution analyzer (Malvern Mastersizer 2000, UK) was used to measure particle size and size distribution of polymer beads with ethanol as dispersant. The median particle size and microsphere surface area per unit volume (S.P.Area) were adopted to characterize the particle size. A larger median particle size and a smaller S.P.Area indicated the increase of particle size [20]. Here, in order to better characterize the size distribution of copolymer microspheres, we referred to the knowledge of mathematical statistics and introduce the coefficient of variation $(\mathrm{CV})$ as a parameter:

$$
\mathrm{CV}=\frac{\sigma}{\mu},
$$

where $\sigma$ is the sample standard deviation and $\mu$ is the arithmetic mean of particle size. Coefficient of variation is a statistic that can measure the variation extent of each observed variation and quantifies the width of the distribution function relative to its mean. A larger coefficient of variation indicates that the distribution of the observed variation is less concentrated [21].

2.3. Synthesis of Ion Polymer Beads. The polymer beads were prepared by the inverse suspension polymerization method. The monomer and crosslink agent mixture solution was prepared by fully dissolving $6 \mathrm{~g}$ sodium p-styrene sulfonate (SSS, monomer), predetermined amount of $\mathrm{N}, \mathrm{N}^{\prime}$ methylenebisacrylamide (MBA, crosslink agent), 0.24 g persulfate (initiator), $20 \mathrm{~mL}$ deionized water, and different porogen with $30 \mathrm{~min}$ ultrasound treatment. Then, $0.48 \mathrm{~g}$ different dispersant and $75 \mathrm{~mL}$ liquid paraffin (oil phase) were added to $250 \mathrm{ml}$ four-necked flask with mechanical stirrer and immersed in a thermostatically controlled heating unit with temperature of $50^{\circ} \mathrm{C}$ for $30 \mathrm{~min}$ until the oil phase was fully melted. Then, the monomer and crosslink agent mixture solution was poured into $250 \mathrm{ml}$ four-necked flask equipped with mechanical stirring and reflux condenser under vigor stirring to produce the inverse suspension system. At last, the temperature was raised up to $90^{\circ} \mathrm{C}$ and maintained for 4 hours with agitation to perform polymerization. After polymerization, the polymer beads were filtered out and rinsed off three times with ethanol and deionized water separately to remove the unreacted materials and dried.

The obtained beads were characterized by FT-IR. The characteristic peaks around $1184 \mathrm{~cm}^{-1}$ (sulfonate group), 1041 $\mathrm{cm}^{-1}$ (benzene ring), $833 \mathrm{~cm}^{-1}$ (1,4-disubstituted benzene), and $1655 \mathrm{~cm}^{-1}$ (amide group) demonstrated the target product was successfully synthesized.

2.4. Acidification and Esterification Evaluation Procedure. Different from the traditional complex process to change the polymer beads into ion exchange resin through sulfonation [22], the sulfonic groups could form after just immersing the synthesized polymer beads containing sulfonate groups into concentrated hydrochloric acid for 4 hours at room temperature in our study. After the acidification, the beads were poured slowly into deionized water and washed by deionized water until the effluent was free of acid. This is the pretreatment process of the synthesized resin beads. Then, the beads were completely ready to begin the catalyzing evaluation procedure. The esterification reaction was performed in fournecked round-bottom flask equipped with mechanical stirrer, condenser, and water splitter. The flask was then immersed in a thermostatically controlled heating unit. Acrylic acid and $n$-octanol were added to the reactor with the weight ratio of 1.7:1, accompanied with azeotropic solvent toluene, inhibitor 4-methoxyphenol (0.1 wt $\%)$, and the polymer beads (5 wt $\%$ in dry weight) after pretreatment and then the esterification was performed at the temperature of $110^{\circ} \mathrm{C}$ for $5 \mathrm{~h}$. After the experiment, the catalysts were filtered out from solution and washed by acetone and deionized water three times separately to prepare for the next esterification.

The esterification was traced by gas chromatography. During the reaction, a series of $0.5 \mathrm{~mL}$ reaction solutions 
TABLE 1: Effect of dispersant on sulfur content and ion exchange capacity of polymer beads.

\begin{tabular}{lcccc}
\hline $\begin{array}{l}\text { Combination } \\
\text { dispersant }\end{array}$ & $\begin{array}{c}\text { Tween60:Span60 } \\
(\mathrm{w} / \mathrm{w})\end{array}$ & $\begin{array}{c}\text { S(wt\%) by elemental } \\
\text { analysis }\end{array}$ & $\begin{array}{c}\text { Ion exchange capacity } \\
(\mathrm{mmol} / \mathrm{g})\end{array}$ & $\begin{array}{c}\mathrm{S}^{*} \text { (wt\%) } \\
\text { by titration }\end{array}$ \\
\hline S60 & $0: 10$ & 9.23 & 2.52 & 8.06 \\
ST66a & $2: 8$ & 10.81 & 2.25 & 7.2 \\
ST66b & $4: 6$ & 9.58 & 2.70 & 8.64 \\
ST66c & $5: 5$ & 9.35 & 2.55 & 8.16 \\
ST66d & $6: 4$ & 7.40 & 1.98 & 6.34 \\
ST66e & $8: 2$ & 6.88 & 1.75 & 5.60 \\
T60 & $10: 0$ & 6.01 & 1.85 & 5.92 \\
\hline
\end{tabular}

MBA:15 wt $\%$ of SSS; no porogen.

were taken out at specific time intervals and diluted with methylene chloride to the concentration of $8 \mathrm{wt} \%$. The gas chromatography (GC2014c, Shimadzu Company, Japan) was equipped with a thermal conductivity detector and column (GsBP-5 model) with hydrogen as a carrier gas at $300^{\circ} \mathrm{C}$. During the analysis, all peaks appeared within ten minutes. The esterification conversion is calculated as follows:

$$
\text { Conversion }=\frac{\left(S_{1} / M_{1}\right)}{\left(S_{1} / M_{1}+S_{2} / M_{2}\right)} * 100,
$$

where $S_{1}$ is the peak area of octyl acrylate, $M_{1}$ is the molecular weight of octyl acrylate, $S_{2}$ is the peak area of $n$ octanol, and $M_{2}$ is the molecular weight of $n$-octanol.

\section{Results and Discussion}

3.1. Influence of Dispersant. Dispersant plays a key role in stabilization of polymerization system and subsequently influences the content of monomers in obtained polymer bead and its morphology. For this kind of inverse suspension polymerization, the nonionic dispersant, such as Tween and Span series, was suitable selection based on our previous work [23]. After some pretest, the Tween60 and Span60 were chosen and the effect of their ratio on the product properties was investigated at first.

It could be seen from Table 1 that the sulfur element ratio (S), which represents the $\mathrm{SO}_{3} \mathrm{H}$ group amount, of pure Tween60 as dispersant system (T60) was much lower than that of pure Span60 system (S60), and combination system with 80 wt\% Span60 and 20 wt\% Tween60 (ST66a) showed the largest value. The higher the sulfur content, the higher the sulfonic group content and the better the catalysis ability. This kind of difference reflected the ratio of sodium styrene sulfonate and the crosslink agent in produced polymer bead. The ratio is decided by dispersant type, because different dispersant combination can lead to different SSS content in aqueous solution drop to form polymer bead and further result in different $S$ content in bead. Almost the same trend was observed in ion exchange capacity, except that the maximum value was obtained in the $60 \mathrm{wt} \%$ Span60 and $40 \mathrm{wt} \%$ Tween60 (ST66b) system. The sulfur element ratio can also be calculated from ion exchange capacity, which was listed in Table $1\left(\mathrm{~S}^{*}\right)$. The values of $\mathrm{S}^{*}$ are a little lower than $S$, which is due to the limitation of penetration of

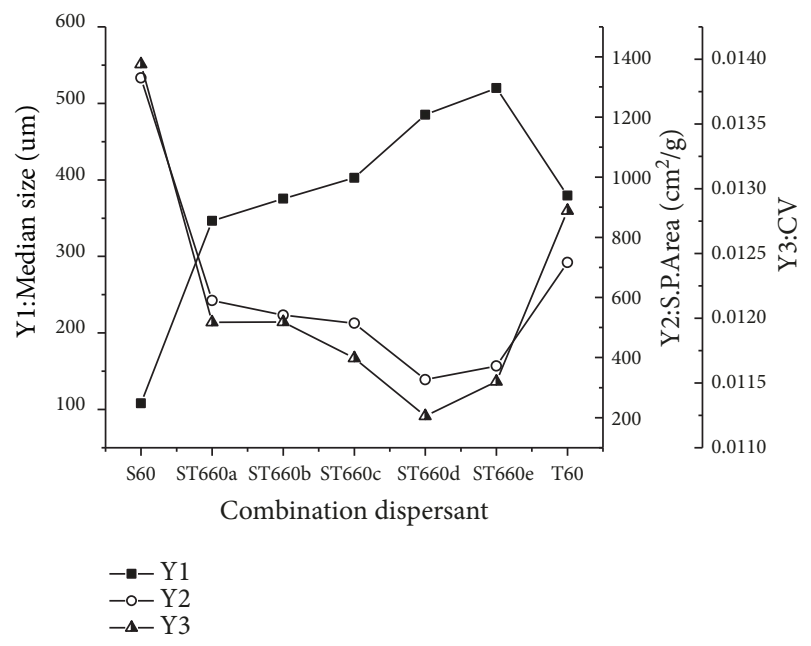

FIGURE 1: Effect of dispersant on polymer beads size and dispersion. MBA:15 wt\% of SSS; no porogen.

alkane aqueous solution into the interior of the polymer bead. However, as the catalyst, ion exchange capacity should be more important than sulfur content, because it directly decided the amount of reactive site and determined the reaction rate.

At the same time, S60 system showed minimum median size (Y1) and maximum S.P. Area (Y2) and CV (Y3), which meant the smallest particle size and largest size dispersion (Figure 1). With the increasing of the Tween60 ratio, the particle size went up then down, but the size dispersion down then up, which could be because higher lipophilic of Span 60 could make the monomer aqueous solution drops in a more stable way and combination dispersant always benefits the suspension polymerization [24].

From the view of catalysis effect, larger surface area per unit volume was always preferable, but too small particle size could make postprocessing and recycling very difficult. So ST66b was selected as optimal dispersant combination to conduct the next step work, due to its moderate particle size, dispersion, the first highest ion exchange ability, and the second highest function group ratio.

3.2. Influence of Posttreatment. Commonly, the posttreatment is just a process to get rid of the unpolymerized 


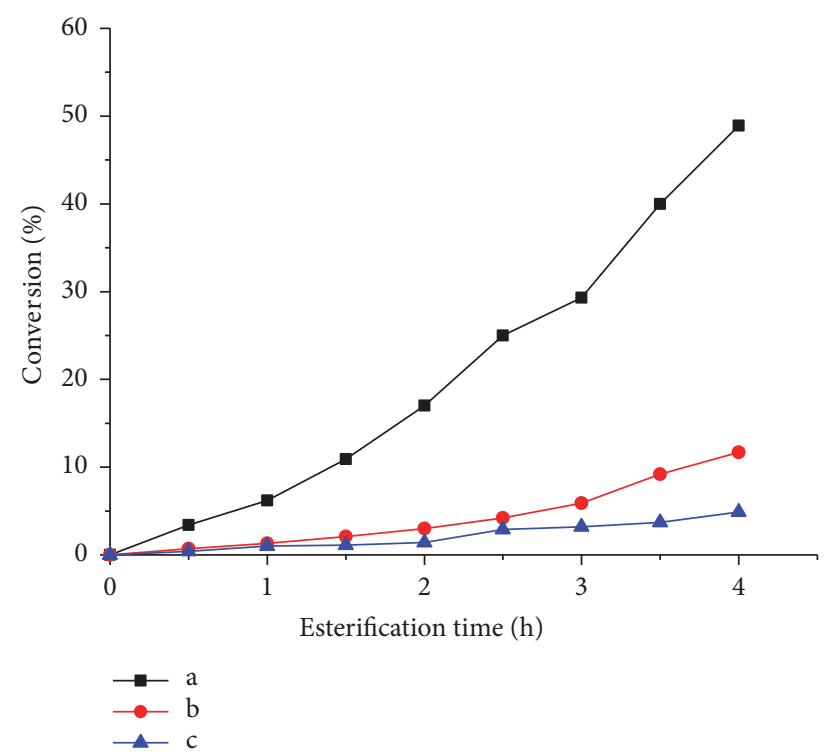

FIGURE 2: Esterification conversion curve with the gel-type beads in swollen form (a); the dried beads with (b) and without (c) acidification process. MBA:15 wt\% of SSS; no porogen.

monomer, dispersant, or other additives. However, this procedure in our investigated system was found to play a very important role for the performance of the polymer beads. Three different methods were compared: the first one was geltype beads obtained through soaking the polymer bead after posttreatment in ionized water for $4 \mathrm{~h}$, the second one was dried beads after posttreatment, and the third one was dried beads before posttreatment.

In Figure 2, curve (a) represented the first method and curve (b) the second method, while curve (c) represented the third one. It could be seen that the polymer beads produced from the first method exhibited the best catalysis effect, around 55\%. The curve of the second method showed only slightly over $15 \%$ conversion, and the unacidified one gave just about $6 \%$ result. It was very easy to understand the trend of curve c, because of lack of proton generation ability. The difference between the first and second method could be attributed to two factors, morphology and acidity.

The first method could make the polymer bead form bigger and looser structure, because both the monomer and crosslink agent are hydrophilic and can absorb and maintain lots of water. It could make acid, alcohol, and solvent penetrate inside of the beads and contact with catalysis site more easily. The adsorbed water can be moved out of the system by water splitter and will not affect the esterification. However, the dry beads from the second method were difficultly swollen by the acid, alcohol, or ester and would keep tight state in the esterification process, which limited its catalysis ability. This was proved by the photographs of two different kinds of polymer beads in Figure 3. The diameter of the first method product is almost 8 times larger than the second one.

Furthermore, as M. Hart's work has shown, when resins are used as dry state, changing the structural features such as the presence of sulfone bridges, the degree of disubstitution
TABLE 2: Effect of crosslink agent content on sulfur content and ion exchange capacity of polymer beads.

\begin{tabular}{cccccc}
\hline & $\begin{array}{c}\text { Crosslink } \\
\text { agent } \\
(\text { wt\% of SSS) }\end{array}$ & \multicolumn{2}{c}{ S (wt\%) } & \multicolumn{2}{c}{$\begin{array}{c}\text { Ion exchange } \\
\text { capacity } \\
\text { (mmol/g) }\end{array}$} \\
& measured & normalized & measured calculated \\
\hline C10 & 9.09 & 10.38 & 11.42 & 3.25 & 0.31 \\
C15 & 13.04 & 9.54 & 10.97 & 2.70 & 0.28 \\
C20 & 16.67 & 8.92 & 10.70 & 2.40 & 0.27 \\
C25 & 20.00 & 8.28 & 10.35 & 2.25 & 0.27 \\
C30 & 23.08 & 7.03 & 9.14 & 1.87 & 0.27 \\
\hline
\end{tabular}

$8 \mathrm{wt} \%$ ST66b; no porogen.

could increase acid strength [17]. But while in the state of swelling in water, the sulfonic content could form internal solution inside the polymer beads, which would increase the acid strengths because of the forming of hydronium ion. So the second method would be adopted in the further work.

3.3. Influence of Crosslink Agent. The crosslink agent might improve crosslink density and lead to high mechanical strength, good heat resistance, and high duration [25], but at the same time, it could bring some other shortcomings. The choice of crosslink agent amount would have a great impact on the final properties of polymer beads.

$\mathrm{N}, \mathrm{N}^{\prime}$-methylenebisacrylamide, one difunctional monomer, was taken as crosslink agent and would influence the density, structure, and morphology of polymer beads. With the ratio of $\mathrm{MBA}$ increased, it was easily understood that the sulfur content (S\%) and ion exchange capacity would decrease due to the concentration effect (Table 2). But normalized S\% value, obtained with measured S\% divided by the corresponding monomer weight percentage, revealed that the increase of crosslink agent indeed declined the monomer conversion and subsequently decreased the ion exchange capacity more remarkably. While the crosslink agent ratio increased from $\mathrm{C} 10$ to $\mathrm{C} 30$, the S\% went down about $32 \%$, but the normalized S\% decreased about $20 \%$, which could not be simply attributed to the concentration effect alone. It was believed that excess crosslink agent would indeed decrease the conversion of sulfonic group containing monomer, because too much crosslink agent could lead its higher ratio in the polymer beads due to its better polymerization activity and the earlier reaching of vitrification point [16].

In Table 2, two different expressions of ion exchange capacity parameters were included. One is measuring ion exchange capacity obtained from titration method, which is the real ion exchange capacity and could represent the catalysis active sites number. Another is calculating ion exchange capacity value, obtained with the value of measured ion exchange capacity divided by the value of measured $\mathrm{S}$, which was used to understand the effect of crosslink agent content on morphology uniformity. The results showed that the measure values decreased reasonably upon the increase of crosslink agent content due to concentration effect. However, the calculated values exhibited very little variation among 


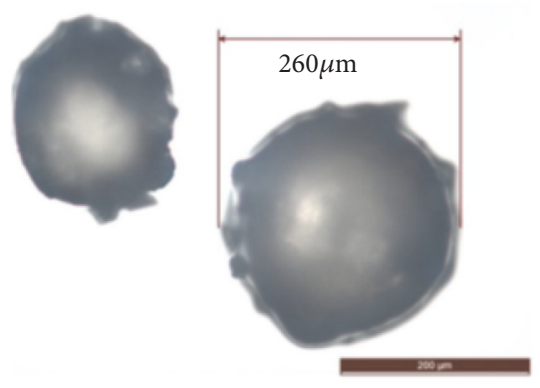

(a)

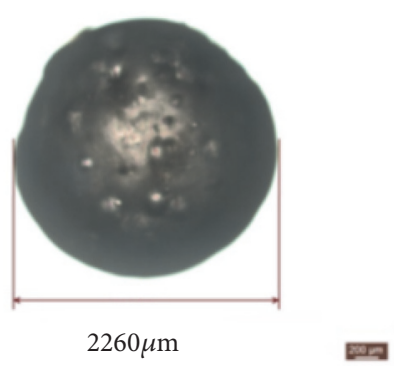

(b)

FIGURE 3: Photographs for polymer beads with different posttreatment (a) with vacuum drying after acidification; (b) without vacuum drying after acidification process (scale bar is $200 \mu \mathrm{m}$ ). $8 \mathrm{wt} \%$ ST66b; [MBA] $=15 \mathrm{wt} \%$ of SSS; no porogen.

TABLE 3: Highest esterification conversion of polymer beads with different crosslink agent content.

\begin{tabular}{lc}
\hline & Highest esterification conversion \\
\hline C10 & $64.7 \%$ \\
C15 & $71.2 \%$ \\
C20 & $95.9 \%$ \\
C25 & $31.3 \%$ \\
C30 & $15.5 \%$ \\
\hline
\end{tabular}

$8 \mathrm{wt} \%$ ST66b; no porogen.

these formulas, except the $\mathrm{C} 10$ sample, which seems just due to the concentration effect. The trend demonstrated that the morphology of polymer beads with different crosslink agent content remained almost the same, because the ion exchange capacity measure result was decided by sulfonic number and the swollen ability of polymer beads in titration solution at the same time.

Light scattering result showed that the particle size gradually decreased with the crosslink agent content increased (Figure 4), and there was platform in C20 and C25 and a sharp decrease to C30 sample. The median size of $\mathrm{C} 10$ sample was about $450 \mu \mathrm{m}$ and that of C30 was about 270 $\mu \mathrm{m}$. But at the same time, the coefficient of variation (CV) curve exhibited a trend of going down and then up, which means that uniformity of polymer beads became better then worse, and gave the best value at C20 sample. It was because too high polymerization rate resulting from excess crosslink agent would lead to the deterioration of inverse emulsion stability and form less uniform smaller particle.

The catalysis ability of this series polymer beads was valued in the synthesis of $n$-octyl acrylate. The results (Table 3 and Figure 5) showed that they all could catalyze the esterification reaction, but the efficiency lied in the broad range. The $\mathrm{C} 10, \mathrm{C} 15$, and C20 exhibited much better catalysis ability, while C25 and C30 were not satisfied. In particular, the C20 sample could reach up to $96 \%$ highest conversion in the first cycle and showed very good reusability. The conversion in the fifth cycle can still achieve more than $80 \%$, which is much better than the first cycle exhibition of commercial catalysts resin Amberlyst 15 (78\%) [26] in acetic acid and $n$-butanol system. In addition, the conversion of C20 was also much

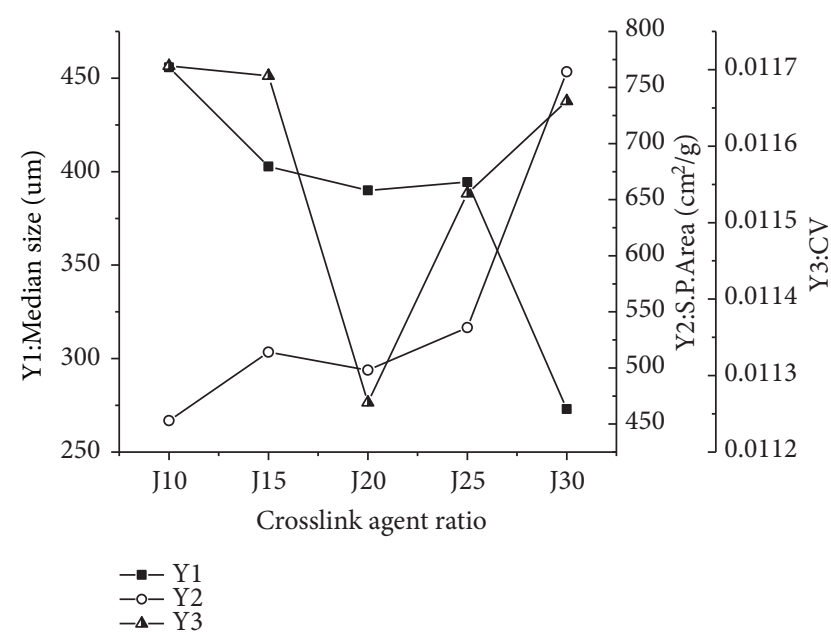

Figure 4: Median size, CV, and S.P. Area of polymer beads with different crosslink agent ratio. $8 \mathrm{wt} \%$ ST66b; no porogen.

better than some other ion exchange resin for different aidalcohol systems, such as Dowex 50WX (35.0\%, acrylic acid with ethanol) [8]; Amberlyst 131 (43.4\%, acrylic acid with $n$ butanol) [11]; Indion 130 (68.2\%, acetic acid with methanol) [27]; Dowex 50 WX2 (70.1\%, acetic acid with isobutanol) [28]; PDVB-0.1- $\mathrm{SO}_{3} \mathrm{H}$ (88.2\%, hexanoic acid with ethanol) [29]. The decrease of conversion upon cycles increasing was certainly observed, which is due to the detachment of sulfonic group in esterification, some product gelling to block the pores of the polymer bead, and some broken beads.

The excellent property of C20 could be attributed not only to its second highest sulfur content, median particle size, and best size distribution, but also to the good crosslink density resulting from the proper crosslink agent concentration. And further, we believed that it should have relationships with the morphology and inner structure [30]. The morphology and topography of the beads were observed by SEM. The spherical morphology of the resin beads could be seen in Figure 6, while there were some small holes on the surface of the polymer beads. It could be explained that no or very little crosslinked polymers could be washed away by deionized water to form the pores in the process of posttreatment. It would be beneficial to improve the effective surface area of 


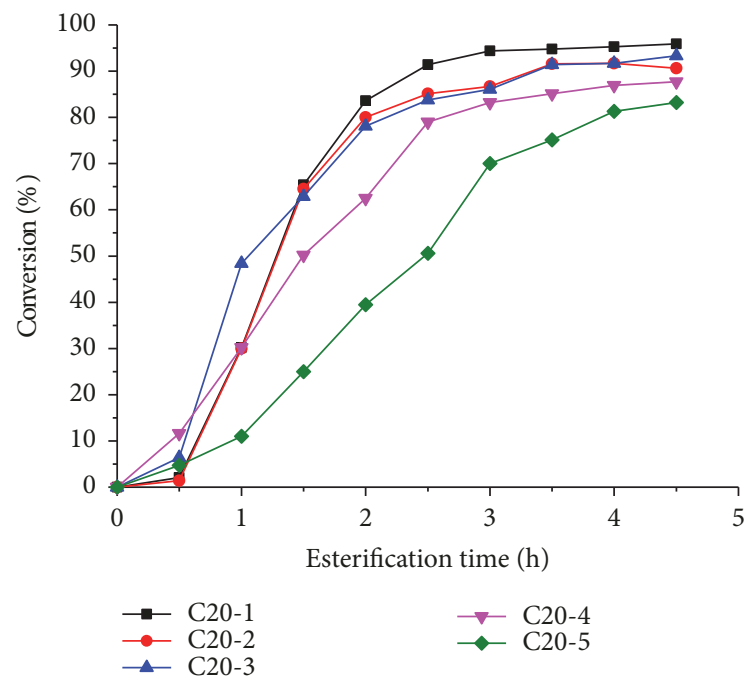

FIGURE 5: Esterification conversion curves with C20 polymer beads at different cycles. $8 \mathrm{wt} \%$ ST66b; no porogen.

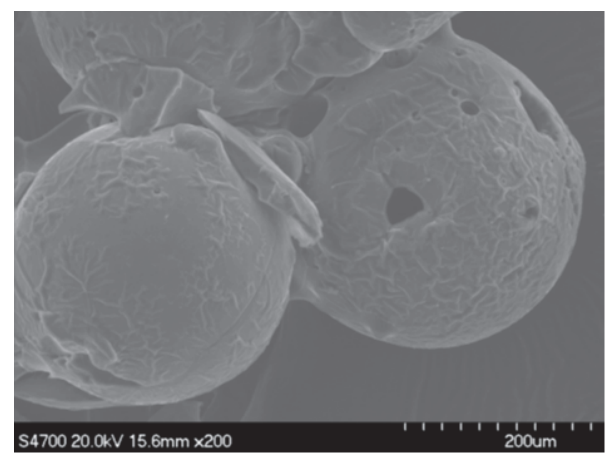

FIGURE 6: SEM micrograph of C20 polymer beads. $8 \mathrm{wt} \%$ ST66b; no porogen.

polymer beads, so that acid, alcohol, and ester in esterification reaction could contact with catalyst adequately to achieve sufficient catalysis, which was not observed for other formulas.

3.4. Effect of Porogen. Furthermore, other six different alcohol compounds, propanediol, n-butanol, 1,4-butanediol, and three different molecular weight polyethylene glycols, were chosen as porogen to investigate their effect on the properties on catalyzing ability, because the pore size and porosity were usually controlled by three experimental parameters: porogen types, amount of porogen, and crosslink density [31].

The first three porogens in Table 4 were short carbon chain alcohols with one hydroxyl group for P2 and two hydroxyl groups for P1 and P3. The last three were polyethylene glycol with different length of carbon chain for two hydroxyl groups. It was shown that $\mathrm{P} 2$ has the maximum sulfur content and ion-exchange capacity, which were bigger, while the rest of porogen appeared to be smaller than that without porogen, C20. It meant that only $n$-butanol could improve the polymerization reaction, due to its lowest solubility in water which makes it have almost no influence on the stability of liquid drop. And at the same time, P2 also
TABLE 4: Effect of different porogen on polymer beads.

\begin{tabular}{lccc}
\hline Porogen & $\begin{array}{c}\text { S\% (wt\%) by } \\
\text { elemental analysis }\end{array}$ & $\begin{array}{c}\text { Ion exchange } \\
\text { capacity } \\
\text { (mmol/g) }\end{array}$ \\
\hline P1 & propanediol & 7.48 & 1.53 \\
P2 & n-butanol & 10.15 & 2.82 \\
P3 & 1,4-butanediol & 8.28 & 1.85 \\
P4 & PEG200 & 8.43 & 2.55 \\
P5 & PEG400 & 8.70 & 1.95 \\
P6 & PEG600 & 7.44 & 1.65 \\
\hline
\end{tabular}

$8 \mathrm{wt} \%$ ST66b; MBA:20 wt $\%$ of SSS.

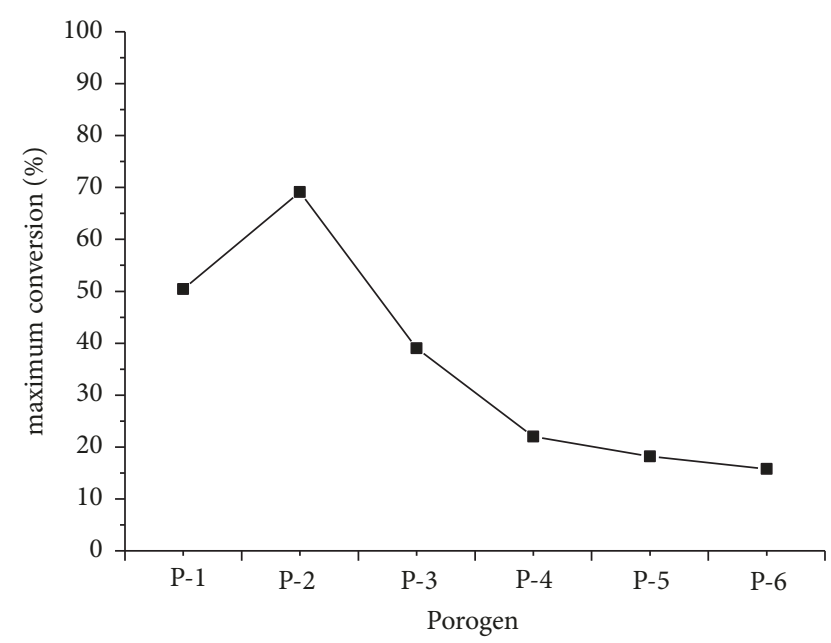

Figure 7: Maximum esterification conversion of polymer beads with different porogens. $8 \mathrm{wt} \%$ ST66b; MBA: $20 \mathrm{wt} \%$ of SSS.

exhibited the highest catalysis ability (Figure 7), which was not only caused by the difference in ion-exchange capacity but also believed to have something with their size and morphology.

It was found that the median size and S.P. Area of P2 (Figure 8) showed just medium value, which implied that particle size was not the main reason for their catalysis performance. Further, the morphology results could give a satisfied insight of this phenomenon. The polymer beads could be found with lots of pores on surface (Figure 9(a)) and holes inside the beads (Figure 9(b)), which would certainly increase the effective catalysis surface. But other systems, taking P5 as an example (other four porogens showed similar results), showed different structure, which was only one center hole and smooth external and internal surface in Figure 9(c). This could also be attributed to the different solubility of porogen in water; better solubility could lead to existence as solution and not take effect as porogen.

But it should be addressed that the catalysis ability of these samples using porogen system was still less than that of C20, which might be due to their mechanical strength. It can be clearly seen from the SEM pictures that some holes have collapsed. So, how to select the better porogen and investigating their mechanism would be the next work. 


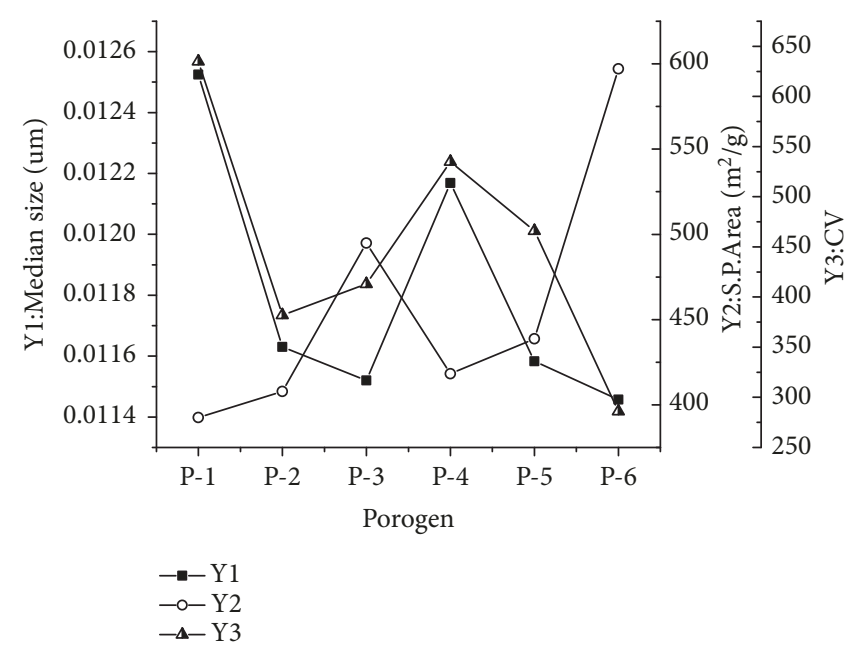

FIGURE 8: Median size, CV, and S.P. Area of polymer beads with different porogens. $8 \mathrm{wt} \%$ ST66b; MBA: $20 \mathrm{wt} \%$ of SSS.

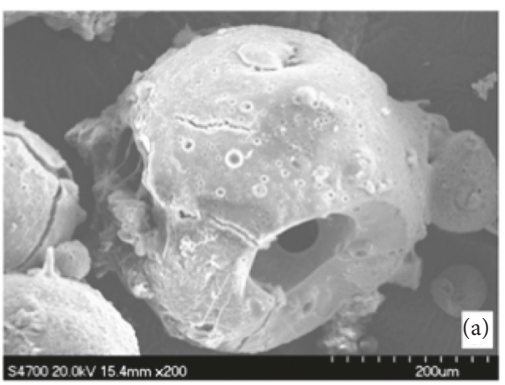

(a)

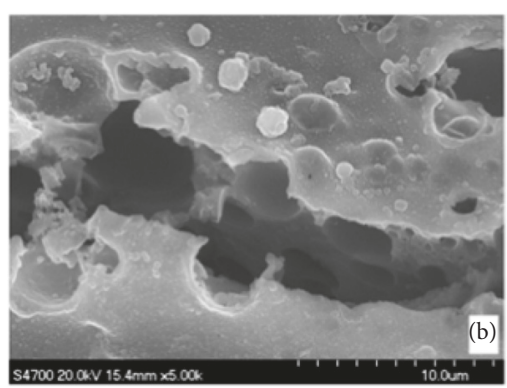

(b)

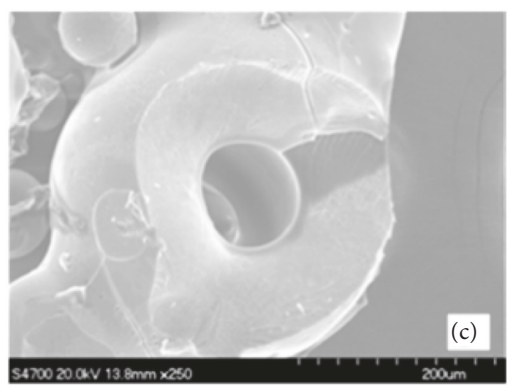

(c)

FIGURE 9: SEM micrographs of P-2 with magnification in 200 (a), 5000 (b), and P-5 with magnification in 250 (c). 8 wt\% ST66b; MBA: 20 wt $\%$ of SSS.

\section{Conclusions}

In this work, we synthesized the ion exchange polymer beads catalyst containing sulfonic group for esterification reaction by inverse suspension polymerization. The combination of Tween 60 and Span60 with the proportion of 4:6 was selected as optimal dispersant system for its moderate particle size and dispersion, best function group ratio, and ion exchange ability. Keeping polymer beads in swollen state was proved to be better posttreatment to form catalyst because of the possibility of formation inner acid solution in esterification process. C20, with $20 \mathrm{wt} \%$ crosslink agent, showed best catalysis ability and repeating properties, due to high sulfur content, median particle size, best size distribution, and forming of pores. It could reach up to $96 \%$ highest conversion and be reused up to 5 times and still achieve $80 \%$ conversion, which was even better than some commercial catalyst. Butanol was the best porogen among the investigated systems due to its lowest solubility in water, but its catalysis ability was less than C20 because of lower mechanical characters.

\section{Data Availability}

The data used to support the findings of this study are available from the corresponding author upon request.

\section{Conflicts of Interest}

The authors declare no competing financial interest.

\section{Acknowledgments}

The authors thank the National Key Research and Development Program of China (2017YFB0307800) and National Natural Science Foundation of China (51373015 and 51573011) for their financial support.

\section{References}

[1] X. Wang, R. Liu, M. M. Waje et al., "Sulfonated ordered mesoporous carbon as a stable and highly active protonic acid catalyst," Chemistry of Materials, vol. 19, no. 10, pp. 2395-2397, 2007.

[2] K. Tanabe and W. F. Holderich, "Industrial application of solid acid-base catalysts," Applied Catalysis A: General, vol. 181, no. 2, pp. 399-434, 1999.

[3] A. Corma and H. García, "Lewis acids: from conventional homogeneous to green homogeneous and heterogeneous catalysis," Chemical Reviews, vol. 103, no. 11, pp. 4307-4366, 2003.

[4] T. Okuhara, "Water-tolerant solid acid catalysts," Chemical Reviews, vol. 102, no. 10, pp. 3641-3666, 2002. 
[5] C. Tagusagawa, A. Takagaki, S. Hayashi, and K. Domen, "Efficient utilization of nanospace of layered transition metal oxide $\mathrm{HNbMoO}_{6}$ as a strong, water-tolerant solid acid catalyst," Journal of the American Chemical Society, vol. 130, no. 23, pp. 7230-7231, 2008.

[6] M. A. Harmer and Q. Sun, "Solid acid catalysis using ionexchange resins," Applied Catalysis A: General, vol. 45, p. 221, 2011.

[7] S. Chang and J. Shaw, "Biocatalysis for the production of carbohydrate esters," New Biotechnology, vol. 109, p. 26, 2009.

[8] G. Jyoti, A. Keshav, J. Anandkumar, and S. Bhoi, "Homogeneous and heterogeneous catalyzed esterification of acrylic acid with ethanol: reaction kinetics and modeling: kinetics and modeling of esterification of acrylic acid with ethanol," International Journal of Chemical Kinetics, vol. 50, no. 5, pp. 370-380, 2018.

[9] A. Chakrabarty and M. M. Sharma, "Cationic ion exchange resins as catalyst," Reactive Polymers, vol. 20, no. 1-2, pp. 1-45, 1993.

[10] M. M. Sharma, "Some novel aspects of cationic ion-exchange resins as catalysts," Reactive and Functional Polymers, vol. 26, no. 1-3, pp. 3-23, 1995.

[11] E. Sert, A. D. Buluklu, S. Karakus, and F. S. Atalay, "Kinetic study of catalytic esterification of acrylic acid with butanol catalyzed by different ion exchange resins," Chemical Engineering and Processing: Process Intensification, vol. 73, pp. 23-28, 2013.

[12] K. A. Kun, R. Kunin, and J. Polym, "Macroreticular resins. III. Formation of macroreticular styrene-divinylbenzene copolymers," Journal of Polymer Science Part A-1: Polymer Chemistry, vol. 6, no. 10, pp. 2689-2701, 1968.

[13] V. M. de-Aguiar, A. L. F. de-Souza, F. S. Galdino, M. M. C. daSilva, V. G. Teixeira, and E. R. Lachter, "Sulfonated poly(divinylbenzene) and poly(styrene-divinylbenzene) as catalysts for esterification of fatty acids," Renewable Energy, vol. 114, pp. 725732, 2017.

[14] M. A. Malik, S. W. Ali, and I. Ahmed, "Sulfonated styrenedivinybenzene resins: optimizing synthesis and estimating characteristics of the base copolymers and the resins," Industrial \& Engineering Chemistry Research, vol. 49, no. 6, pp. 2608-2612, 2010.

[15] S. Kiatkamjornwong and P. Phunchareon, "Influence of reaction parameters on water absorption of neutralized poly(acrylic acid-co-acrylamide) synthesized by inverse suspension polymerization," Journal of Applied Polymer Science, vol. 72, no. 10, pp. 1349-1366, 1999.

[16] C. Mayoux, J. Dandurand, A. Ricad, and C. Lacabanne, "Inverse suspension polymerization of sodium acrylate: synthesis and characterization," Journal of Applied Polymer Science, vol. 77, no. 12, pp. 2621-2630, 2000.

[17] M. Hart, G. Fuller, D. R. Brown, J. A. Dale, and S. Plant, "Sulfonated poly(styrene-co-divinylbenzene) ion-exchange resins: acidities and catalytic activities in aqueous reactions," Journal of Molecular Catalysis A: Chemical, vol. 182-183, pp. 439-445, 2002.

[18] T. R. Theodoro, J. R. Dias, J. L. Penariol, J. OV. Moura, and L. G. Aguiar, "Sulfonated poly (styrene-co-ethylene glycol dimethacrylate) with attractive ion exchange capacity," Polymers for Advanced Technologies, vol. 29, no. 11, pp. 2759-2765, 2018.

[19] W. B. Ying, J. U. Jang, M. W. Lee, T. S. Hwang, K. J. Lee, and B. Lee, "Novel flexible styrenic elastomer cation-exchange material based on phenyl functionalized polystyrene-butadiene copolymer," Journal of Industrial and Engineering Chemistry, vol. 128.9, p. 47, 2017.

[20] P. Liang, Z. Jiang, Z. Meng, J. Nie, and Y. He, "Investigation of stabilizer-free dispersion polymerization process of styrene and maleic anhydride copolymer microspheres," Journal of Polymer Science Part A: Polymer Chemistry, vol. 48, no. 24, pp. 56525658, 2010.

[21] Z. Sun, "Study on usage of coefficient of variation for statistics of magnetic parameters of samples," Geology \& Exploration, vol. 65, p. 45, 2009.

[22] C. Martin and J. Cuellar, "Synthesis of a novel magnetic resin and the study of equilibrium in cation exchange with amino acids," Industrial \& Engineering Chemistry Research, vol. 43, no. 2, pp. 475-485, 2004.

[23] F. P. Wu, M. Q. Shi, Y. L. Zhang, Y. X. Zhang, and Y. He, "Nano water-soluble microgel oil displacement material and preparation method," ZL200510012255.0, 2005.

[24] E. Vivaldo-Lima, P. E. Wood, and A. E. Hamielec, "An updated review on suspension polymerization," Industrial \& Engineering Chemistry Research, vol. 36, no. 4, pp. 939-965, 1997.

[25] C. A. Toro, R. Rodrigo, and J. Cuellar, "Sulfonation of macroporous poly(styrene-co-divinylbenzene) beads: effect of the proportion of isomers on their cation exchange capacity," Reactive and Functional Polymers, vol. 68, no. 9, pp. 1325-1336, 2008.

[26] J. Gangadwala, S. Mankar, and S. Mahajani, "Esterification of acetic acid with butanol in the presence of ion-exchange resins as catalysts," Industrial \& Engineering Chemistry Research, vol. 42, no. 10, pp. 2146-2155, 2003.

[27] P. E. JagadeeshBabu, K. Sandesh, and M. B. Saidutta, "Kinetics of esterification of acetic acid with methanol in the presence of ion exchange resin catalysts," Industrial \& Engineering Chemistry Research, vol. 50, no. 12, pp. 7155-7160, 2011.

[28] A. Izci and F. Bodur, "Liquid-phase esterification of acetic acid with isobutanol catalyzed by ion-exchange resins," Reactive and Functional Polymers, vol. 67, no. 12, pp. 1458-1464, 2007.

[29] F. J. Liu, X. Meng, Y. L. Zhang, L. M. Ren, F. Nawaz, and F. S. Xiao, "Efficient and stable solid acid catalysts synthesized from sulfonation of swelling mesoporous polydivinylbenzenes," Journal of Catalysis, vol. 271, no. 1, pp. 52-58, 2010.

[30] M. A. Tejero, E. Ramírez, C. Fité, J. Tejero, and F. Cunill, "Esterification of levulinic acid with butanol over ion exchange resins," Applied Catalysis A: General, vol. 517, pp. 56-66, 2016.

[31] W. L. Sederel and G. J. De Jong, "Styrene-divinylbenzene copolymers. Construction of porosity in styrene divinylbenzene matrices," Journal of Applied Polymer Science, vol. 17, no. 9, pp. 2835-2846, 1973. 


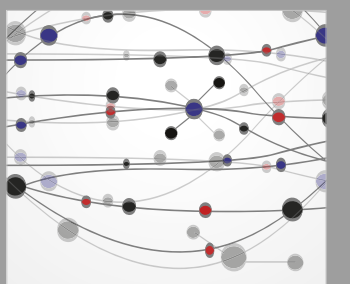

The Scientific World Journal
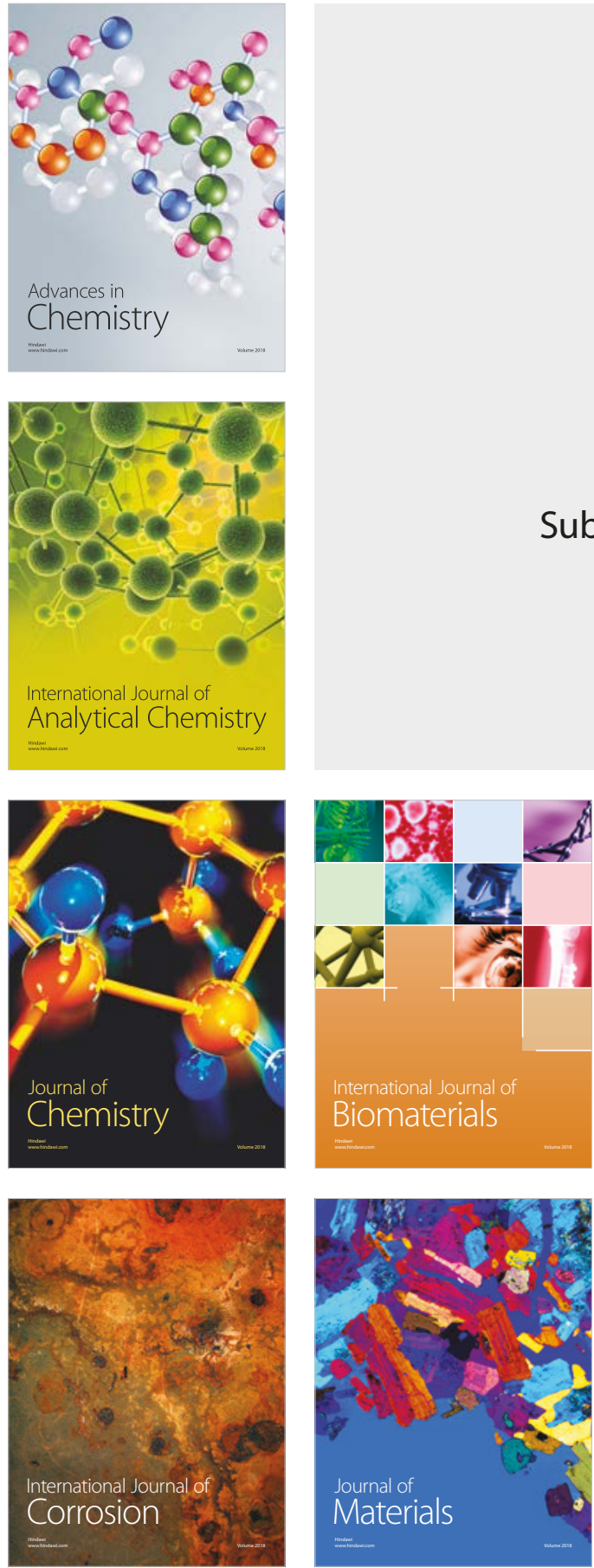

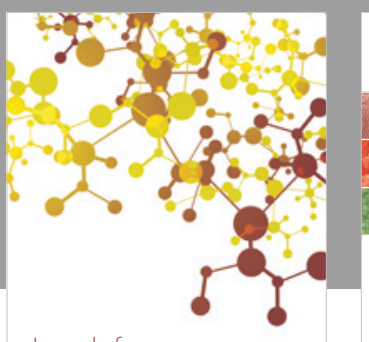

Journal of

Applied Chemistry
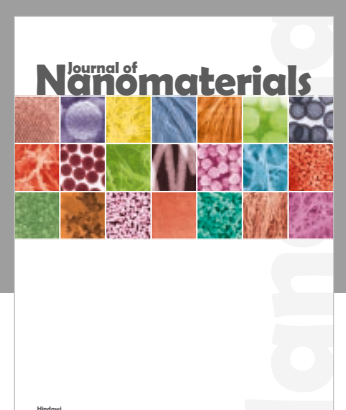

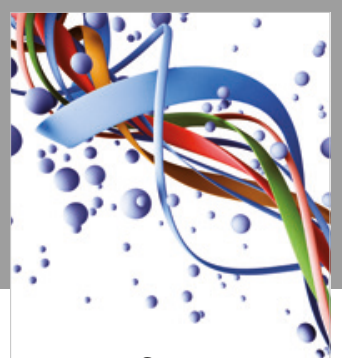

Scientifica

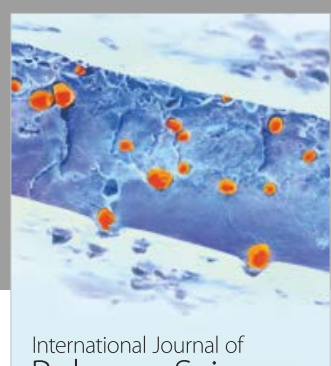

Polymer Science

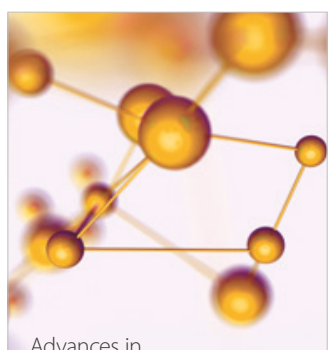

Physical Chemistry
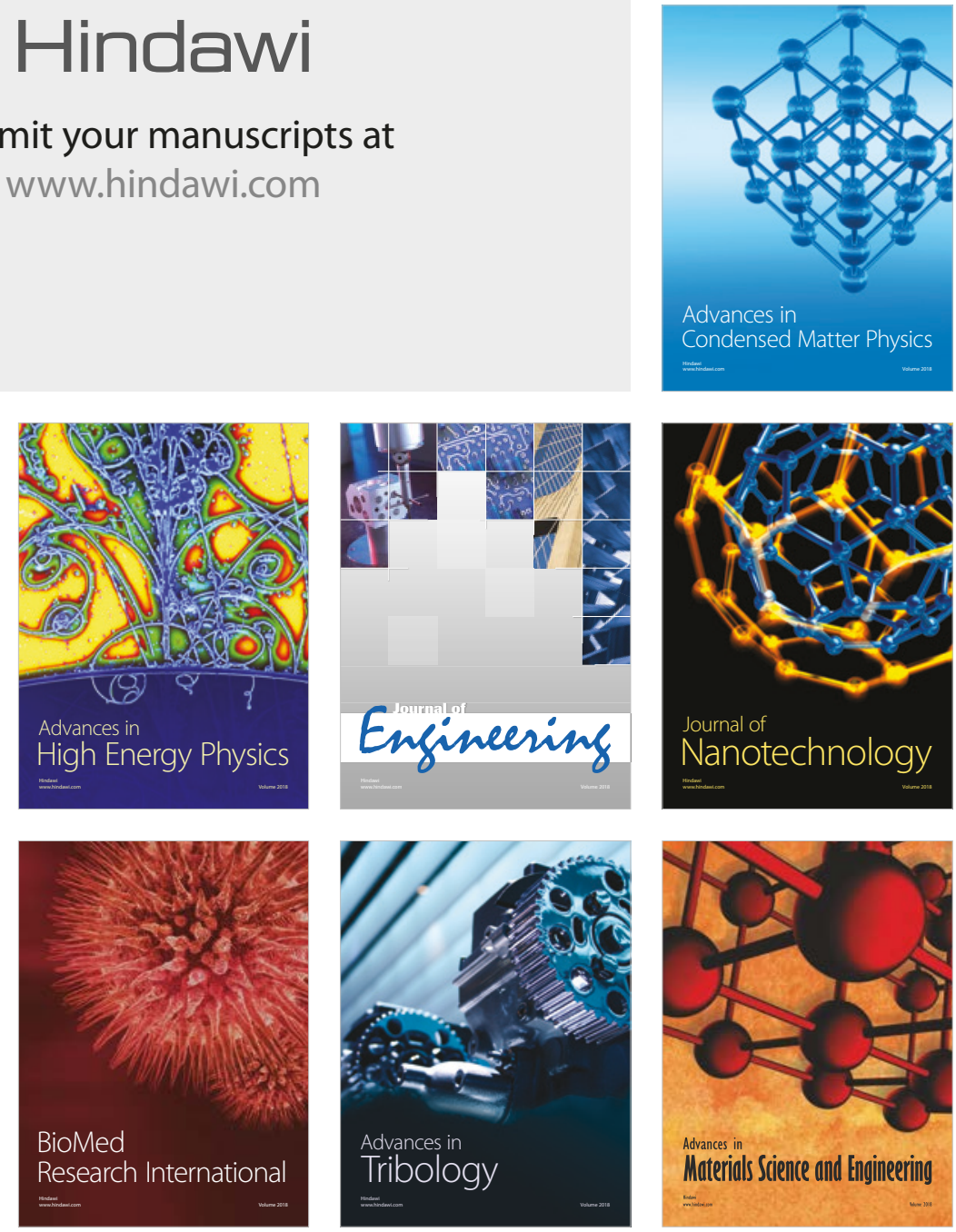